\title{
Dinámicas de racismo en el Perú: la perspectiva cultural de Gonzalo Portocarrero
}

\author{
Víctor Vich*
}

* Víctor Vich es doctor en Literatura Hispanoamericana por Georgetown University. Fue miembro de Comité Directivo del Consejo Latinoamericano de Ciencias Sociales - Clacso (2007-2009) y del equipo curatorial del Lugar de la Memoria en el Perú. Actualmente, es director de la Maestría en Estudios Culturales en la Pontificia Universidad Católica y profesor de la Escuela Nacional Superior de Bellas Artes en el Perú. Correo electrónico: vvich@pucp.pe

Fecha de recepción: 26/06/2019. Fecha de aceptación: 08/08/2019 


\section{Dinámicas de racismo en el Perú: la perspectiva cultural de Gonzalo Portocarrero}

\section{RESUMEN}

Gonzalo Portocarrero construyó tres metáforas para explicar las dinámicas del racismo en el Perú, vale decir, para intentar describir su modo de funcionamiento y la manera particular en la que este se había producido (y asentado) en las prácticas sociales y en el imaginario existente. Las tres metáforas fueron las siguientes: el fundamento invisible, el fantasma del patrón y la utopia del blanqueamiento. Este ensayo explica cada una de ellas y las hace dialogar con algunos de los principales aportes que, para entenderlo, se han propuesto en el Perú y en la teoría crítica actual.

Palabras clave: Gonzalo Portocarrero, racismo, metáforas, Perú.

\section{Dynamics of racism in Peru: the cultural perspective of Gonzalo Portocarrero}

\section{Abstract}

Gonzalo Portocarrero constructed three metaphors to explain the dynamics of racism in Peru, that is, to try to describe its mode of operation and the particular way in which it had been produced (and established) in social practices and in the social imaginary. The three metaphors were the following: the invisible foundation, the ghost of the patron and the utopia of whitening or "blanqueamiento". This essay explains each of them and brings them into dialogue with some of the main contributions that, in order to understand it, have been proposed in Peru and in current critical theory.

Keywords: Gonzalo Portocarrero, racism, metaphors, Peru. 
En realidad, cuanto más ahondamos en nuestro presente tanto más nos encontramos con nuestro pasado. Nos sigue acompańando la realidad de un país fragmentado por las exclusiones y odios, donde la violencia es el fundamento de un orden precario, constantemente acechado por una conflictividad irresuelta. (Portocarrero, 2003, p. 230)

A lo largo de su amplio trabajo, Gonzalo Portocarrero construyó tres metáforas para explicar las dinámicas del racismo en el Perú, vale decir, para intentar describir su modo de funcionamiento y la manera particular en que se había producido (y asentado) en las prácticas sociales y en el imaginario peruano. En sus escritos, el racismo fue entendido como un mecanismo de poder que construye identidades, refuerza relaciones de dominación y permea el funcionamiento de la vida colectiva. El racismo, como sabemos, refiere a un escenario donde se cree que las razas existen, que no todas son iguales, donde las diferencias están siempre jerarquizadas y se constituye como la práctica de mirar al otro como inferior, como desigual o carente de algo. Estudiarlo implicaba entonces observar cómo se clasifica a la población, cómo se jerarquiza a las personas y cómo se asume que unos son «superiores» o «inferiores». De manera muy clara, sostuvo que las prácticas racistas siguen actuando en el Perú de hoy.

Portocarrero partió de constatar que el Perú nunca se imaginó a sí mismo como una comunidad de iguales. La promesa republicana fue incapaz de constituir una nación (y un Estado) que garantizara la vida igual para todos: las herencias del pasado, los intereses particulares y la falta de un verdadero sentido de comunidad fueron entedidas como trabas que, según su opinión, han impedido el verdadero desarrollo de los ciudadanos y de la comunidad en su conjunto. Pensó entonces que muchas prácticas actuales podían conectarse con aquellas que se instalaron durante la colonia a pesar de todos los cambios y transformaciones existentes. No se trató, sin embargo, de entender al racismo como algo inmutable y estático, sino como un patrón de poder que ha ido encontrando formas cada vez más complejas de manifestarse.

Podemos decir que, en sus escritos, el racismo aparece como una mentalidad colectiva y como una práctica. $\mathrm{O}$, mejor dicho, como una práctica que corresponde con un conjunto de representaciones. Como mentalidad, como imaginario o, incluso, como ideología, el racismo se dedica a producir estereotipos, fantasmas y miedos. Como práctica, contribuye a estructurar buena parte de las relaciones sociales y supone la articulación de un acto de diferenciación y de exclusión social.

Los rasgos físicos, empezando por el color de piel, son hechos muy importantes. Funcionan como signos de diferencias sociales y los interpretamos gracias a los códigos aprendidos en la infancia. De hecho, estos rasgos son objeto de valoraciones estéticas que califican a las personas que los poseen (Portocarrero, 2007, pp. 181-182). 
Portocarrero partió por entender al racismo como un problema de mentalidades relativo al carácter de sociedades poscoloniales que no han logrado descolonizar su imaginario y donde los fantasmas del pasado acosan permanentemente al presente. En ese sentido, sostuvo que el racismo era un problema afianzado en el inconsciente social. «Se puede decir que todos los seres humanos somos colonizados, pues, querámoslo o no, nuestra socialización está presidida por ciertos mandatos que no podemos dejar de interiorizar y a los que tenemos que responder» (2015a, p. 45). El racismo «existe en lo privado» (2007, p. 185) y su ámbito de reproducción es la familia (1992, p. 187).

Sin embargo, Portocarrero entendía al racismo no solo como un problema de mentalidades, sino que también intentó cartografiarlo como un conjunto de prácticas sociales. Más que en su contenido, lo central del racismo se ubica en lo que hace y en el poder que ejerce. La jerarquización y la consecuente legitimación de la desigualdad se presentan como prácticas hondamente asentadas en el Perú y como parte de un sistema de dominación mayor. Portocarrero intentó pensar de qué forma el racismo es socialmente reproducido y cómo continúa modelando el comportamiento de los ciudadanos y de las instituciones sociales. Desde ahí, puede decirse que no lo entendió tanto como una ideología sino, más bien, como una «práctica ideológica» cuyo contenido se adapta y cambia de acuerdo a nuevos contextos históricos (Wetherell y Potter, 1992; Zavala y Back, 2017). En ese sentido, el racismo permite describir un conjunto de dinámicas de funcionamiento de la vida peruana en general.

Las tres metáforas a partir de las cuales explicó el racismo peruano fueron las siguientes: el fundamento invisible, el fantasma del patrón y la utopía del blanqueamiento. Portocarrero pensaba que las metáforas no son patrimonio único de la literatura sino que resultan decisivas para describir sociedades cuya densidad nunca se deja capturar por un conjunto de «datos» que nunca consiguen capturar completamente la complejidad de dinámicas de lo social. Sostenía que era necesario contar con nuevos instrumentos que capturen su densidad y contradicción y, para él, las metáforas cumplían esa función. Se trataba, entonces, de ir más allá de lo taxonómico para moverse hacia lo histórico y lo metafórico como encuadres fundamentales del conocimiento en general. Voy a intentar explicar cada una de estas metáforas y las haré dialogar, muy brevemente, con algunos de los principales aportes que, para entender el problema del racismo, se han propuesto en el Perú y en la teoría crítica actual.

\section{EL «FUNDAMENTO INVISIBLE»}

Como hemos afirmado ya, Portocarrero insistió en que el racismo fue la ideología del Estado oligárquico (1895-1968) y que ha sido uno de los mayores obstáculos que 
ha encontrado la constitución democrática en el Perú. «El proyecto nacional criollo pretendió desconocer la tradición indígena y marcar la colectividad peruana con su sello señorial y trasgresor» sostuvo enfáticamente (2015a, p. 7). Como sabemos, durante mucho tiempo, el indio fue representado como un otro amenazador frente al cual había que protegerse o excluir. Desde ahí, el racismo se instaló como un modo de interacción social que permitió que las élites fortalecieran un sentimiento de superioridad y se constituyó como «uno de los grandes obstáculos para consolidar una identidad nacional» (1992, p. 188). Esta misma idea también ha sido sostenida por otros investigadores como Nelson Manrique: «El racismo anti-indígena fue el principal tipo de discriminación en el Perú hasta mediados del siglo XX» (2014, p. 67).

En el Perú, en efecto, dominó una visión del país en la que se despreciaba todo lo que no fuera blanco. Clemente Palma, por ejemplo, planteó que la «raza» era una especie de "patrimonio genético» en el que supuestamente podían localizarse las posibilidades de progreso de un pueblo. Sostuvo que a cada raza le correspondía una función en la división del trabajo: a los negros les tocaba la servidumbre y a los blancos ser la autoridad. Según Portocarrero, en los escritos de Clemente Palma el Perú parece "un país condenado por la biología, presa de fatalidad y sin salida» (1995, p. 227). En ese sentido, la república peruana se fundó, entonces, no como un proyecto de iguales, sino bajo una división de razas y clases o, más precisamente, de «clases racializadas» (Quijano, 2000). Como discurso, el racismo justificó la exclusión de la mayoría de la población en el manejo de lo público. Como práctica, jerarquizó, discriminó y explotó: se fue asentando como un dispositivo adicional en la estructuración de las relaciones sociales.

Por «fundamento invisible», entendió entonces una interacción de ideologías y prácticas, hondamente asentadas, que actuaban como dispositivos para la interacción social. Para Portocarrero, las prácticas racistas contribuyeron a formar y a constituir al Estado peruano tanto por la desvaloración de las culturas no occidentales como por la inferiorización de personas de «color» (2007, p. 181). En ese sentido, él también hubiera sostenido que «la marginación histórica del indio no debe entenderse como expresión de una falta o falla del Estado-nación peruano, sino como su condición necesaria y constitutiva (Drinot, 2014, p. 44).

Digamos entonces que el racismo se encuentra vinculado a la ausencia de participación de sectores populares en las decisiones del país y tuvo que ver con la falta de una vocación democrática en las élites. La raíz de esta falta se encuentra en un imaginario colonizado y en un conjunto de prácticas discriminatorias que persisten todavía. No se trata, sin embargo, de un racismo ejercido desde los blancos contra mestizos, cholos o indios. En el Perú, todos los ciudadanos se clasifican, se jerarquizan, se estereotipan y se marginan unos con otros. En ese sentido, no se trata de identidades claramente delimitadas sino de "posiciones» que si bien son siempre 
móviles y porosas no dejan de encontrar formas de constituirse rígidamente: Lima/ provincias, ciudad/campo, valle/puna.

Todos manejamos un sistema de clasificatorio de los demás. Inmediatamente que vemos a alguien, lo vamos categorizando, ubicando dentro de cierto espectro de posibilidades, y es en función de esta clasificación que vamos a tratarlo, que vamos a proponerle un patrón de interacción. Y él va a hacer lo propio y nos va a proponer otro patrón de interacción. La apariencia física, la cultura y la clase son los tres fundamentos del repertorio subjetivo de identidades. No necesariamente las tres van en el mismo sentido (1992, p. 189).

La metáfora del "fundamento invisible» refiere entonces a una idea de separación, a la imposibilidad que los peruanos tenemos de representarnos como iguales. La raza se concibe como un conjunto de propiedades que distinguen a unos de otros y el prejuicio racial se impone en distintos tipos de interacciones cotidianas. Portocarrero afirmó que es un error entender el racismo como una práctica en vías de extinción pues, por el contrario, este se presenta como el «fundamento invisible» de la modernidad peruana. El racismo es un dispositivo de poder que viene de lejos pero que se resignifica y actúa dentro del marco de la democratización social. Para él, la modernidad no puede ser explicada sin dicho dispositivo que, desde muchas formas, también la constituye y le da forma. El racismo debe entenderse como una especie de «fundamento» porque estructura buena parte de vínculo social y se reproduce en él.

El discurso moderno está mediatizado por la vigencia de un discurso colonial, subterráneo, pero no menos influyente. Se trata del viejo discurso racista donde la dominación étnica y la desigualdad estuvieron reconocidas por las leyes. Este discurso funcionaba a favor de las élites criollas y blancas y el desmedro del mundo indígena. Si los deseos de igualdad y libertad no están internalizados en el Perú actual, ello obedece a la resistencia que suponen otros deseos igualmente presentes. La expectativa de sacar ventaja surge de la posibilidad de conocer al otro, de una visión jerarquizada de la sociedad donde no todos tenemos iguales derechos (2010b, p. 305).

\section{EL «FANTASMA DEL PATRÓN»}

Hemos dicho que más allá de figurar al racismo como un problema únicamente referido a imaginarios sociales, Portocarrero lo entendió también como una práctica, como un ejercicio de autoridad. Desde ahí, era indispensable describir sus estrategias para definir la particularidad de su funcionamiento y mostrar sus contornos.

$\mathrm{Al}$ respecto, su hallazgo fue muy claro: la «cultura de la hacienda» es lo decisivo en la estructuración del racismo en el Perú. La hacienda y el gamonalismo parecen haber sido las instituciones productoras de un conjunto de dinámicas hoy todavía 
presentes. Digamos que, al situar el problema al interior de la hacienda, Portocarrero subrayó el vínculo del racismo con la organización del trabajo y en relación con la explotación económica.

Para él, la "cultura de la hacienda» instauró un tipo de «cultura servil» que, de alguna manera, perdura en la actualidad y que los peruanos seguimos reproduciendo día a día. Como sabemos, luego de la independencia, y durante todo el siglo XIX, la situación de las comunidades indígenas no mejoró significativamente $y$, antes bien, se reforzaron ciertos patrones de exclusión social. A pesar de que el general San Martín llamó a los indios «ciudadanos» y Bolívar promulgó algunas leyes liberales, los tributos indígenas aumentaron y la gran propiedad de la tierra comenzó a expandirse. Para muchos historiadores, fue el siglo XIX, donde la falta de derechos de las comunidades indígenas y la usurpación de tierras terminó por consolidarse.

¿Qué entendía Gonzalo Portocarrero por la "cultura de la hacienda»? A parir de una investigación realizada por Patricia Ruiz Bravo y Eloy Neyra (2001), la respuesta está en la localización de las figuras del "patrón» y del «siervo». Ambas, emergen como identidades centrales en el imaginario social peruano en tanto contribuyen a la construcción de buena parte de las interacciones sociales.

Lo que pretendo proponer es que los ciudadanos (siervos) proyectan en las figuras de autoridad una imagen del patrón despótico que tiene que ver ya más con el pasado que con el presente... Y de otro lado ocurre lo mismo con las autoridades (patrones); es decir, proyectan sobre la ciudadanía la imagen del siervo ignorante y repulsivo (Portocarrero, 2010a, p. 14).

Aspirar a «ser patrón» y posicionar a los demás como «siervos» parece un rasgo típico del funcionamiento de muchas instituciones y relaciones laborales en el Perú. En palabras de Mbembe, la lógica de los patrones ha sido producir «un lazo social de sumisión y un cuerpo siempre expuesto a la voluntad de un amo que se empeña en obtener de él la máxima rentabilidad» (2016, p. 52). Debido a esta dinámica, existe en el Perú un goce particular en el ejerciendo poder en el marco de relaciones sociales donde algunos quieren acapararlo todo y muchos todavía no acaban de sentirse ciudadanos:

Existe pues un círculo vicioso: si nos vemos como víctimas, el otro tendrá que ser déspota, y si percibimos al otro como déspota, entonces seremos sus víctimas. En cualquier caso, tenemos una desconfianza profunda (Portocarrero, 2010a, p. 21).

Sin embargo, su argumento más importante fue el siguiente: sostuvo que es cierto que, luego de la reforma agraria la figura histórica del patrón ha caído, pero el problema es que su «lugar» ha quedado vacío y el problema es que hoy muchos quieren ocuparlo. Desde este punto de vista, la vida peruana aparece como un desesperado intento por ocupar ese lugar (por llegar a "ser patrones») para aprovecharse 
de los demás y ejercer poder. De hecho, en el Perú actual, muchas autoridades siguen tratando a los trabajadores como verdaderos siervos y el problema se vuelve más grave aún "porque lo que anhela el siervo-ciudadano es convertirse en patrónautoridad, en el amo que goza del poder de subyugar a gente como él» (Mbmbe, 2016, p. 23). Dicho de otra manera: muchos de aquellos que han sido discriminados pronto reproducen el racismo con quienes se encuentran en su vieja posición y el resultado es, finalmente, el de «un fuego cruzado de todos contra todos y hasta de uno mismo» (Rochabrún, 2014, pp. 17-18).

Así, se ha instalado en el Perú un juego perverso que trae como consecuencia la extrema fragilidad de los vínculos sociales. «Prima entonces un individualismo trasgresor que socava cualquier principio de autoridad» (2010a, p. 26). De hecho, en el Perú siempre podemos percibir cómo muchas de las autoridades existentes son "patrones encubiertos", vale decir, personas que reproducen la "cultura de la hacienda" y que no se cansan de ejercer un viejo poder sobre los demás.

Las autoridades realmente existentes no son legítimas porque su acción no apunta al cumplimiento de las leyes y derechos de la gente sino, sobre todo, a su beneficio personal. Es decir, ellos no son lo que pretenden. Su investidura legal es solo una mascarada. Detrás del congresista de la república o del rector universitario, están, en realidad las viejas figuras de cacique o patrón (2010a, p. 21).

La subjetividad peruana aparece, pues, extraordinariamente compleja. El siervociudadano contiene dentro de sí la figura del patrón-autoridad, a la que odia pero también ama. En realidad, se trata de una situación de entrampamiento de donde nacen una multitud de actitudes ambiguas: la queja contra la prepotencia pero la fascinación con el autoritarismo, el rechazo a la trasgresión de la ley pero la admiración por el trasgresor, el deseo de ser autoridad pero sin el compromiso con la ley que esta posición implica (2010a, p. 23).

El punto clave radica en notar que, encarnado en este fantasma —el «fantasma del patrón»- el racismo se empalma con el autoritarismo que es, en última instancia, discriminación, anomia y corrupción. La figura del patrón cumplirá, para Portocarrero, una función decisiva en la legitimación de exclusiones sociales pues naturaliza las desigualdades consagrando un orden social en el que los lugares siempre se jerarquizan para ejercer el poder.

\section{LA «UTOPÍA DEL BLANQUEAMIENTO»}

Bajo esta metáfora, Portocarrero trató de sostener que la raza existe mucho más como fantasía cultural que como rasgo genético y sus ideas coincidirían en la necesidad de "trazar una genealogía histórica del racismo en el Perú que conecte el racismo actual con las jerarquías racializadas que se establecieron en la colonia 
(Drinot, 2014, p. 36). Portocarrero enfatizó que el color de la piel está cargado de un conjunto de significados que son históricos y que, por lo mismo, siempre pueden cuestionarse. Más allá de las profundas transformaciones sociales que en las últimas décadas cambiaron el rostro al Perú, afirmó que seguimos viviendo «en una sociedad donde los modelos de belleza no son representativos de la realidad de sus habitantes» (2013, p. 61) y que «aspiramos a ser lo que no somos, pues lo valoramos más» (2015b, p. 47).

Para argumentar esta dinámica, partió de una crítica a la categoría del mestizaje. En su opinión, su uso ha sido ideológico e incompleto. Al interior de un país realmente heterogéneo, el discurso sobre mestizaje ha invisibilizado diferencias de todo tipo. Decir que «todos somos mestizos» oculta los grandes antagonismos sociales y esconde finas dinámicas culturales. «El problema del mestizaje peruano es que se define más como negación (ni blanco ni indio) que como una afirmación» (1992, p. 188), sostuvo con lucidez. Para Portocarrero, debajo del mestizaje, fluye siempre un discurso clasificador. El problema es que en el Perú la gente no es simplemente mestiza, pues «lo que realmente importa socialmente es la composición del mestizaje: la blancura relativa de algunos frente a otros» (2015a, p. 64).

Desde ahí, por «utopía del blanqueamiento», entendió un proyecto transgeneracional de «mejora de la raza» que puede rastrearse en los distintos discursos y prácticas de la escena cultura peruana (2013, p. 62). Recordemos que «nuestra socialización está presidida por ciertos mandatos que no podemos dejar de interiorizar y a los que tenemos que responder» (2015a, p. 45). Sostuvo entonces que uno de esos mandatos consiste en el intento de apartarse de la herencia indígena mediante un conjunto de estrategias que denominó «utopía del blanqueamiento»: dinero, matrimonios, lugares de poder, etc.

La utopía del blanqueamiento reconcilia la tensión entre inmovilidad de la adscripción racial y la fluidez de la estratificación basada en el trabajo y el dinero. Entonces, el sistema resulta expresivo de una sociedad donde la voluntad de jerarquizar convive con la democratización de oportunidades económicas (2013, p. 64).

Se trata, así, de una especie de «resolución» en un contexto cada vez más fluido donde resulta difícil definir y jerarquizar de una manera precisa e indudable. El éxito económico puede, en efecto, «limpiar» el color originario al interior de una realidad social cada vez más móvil e híbrida, pero que siempre esconde algún tipo de tensión social.

Ahora bien, ¿Qué tipos de sujeto produce la «utopía del blanqueamiento»? En principio, un sujeto colonizado que rechaza su condición y que sueña con que su descendencia pueda aumentar su valor social $(2013$, p. 71$)$. Se trata de subjetividades que han internalizado tanto un sistema clasificatorio como la mirada de 
un otro poderoso que insiste en funcionar como arquetipo y modelo. Prisioneros de un fantasma, la «utopía del blanqueamiento» da cuenta de un imaginario todavía colonizado (2013, p. 62).

Si se tiene al frente a una persona más blanca o con más dinero, se va a sentir menos; si es una persona más oscura y de inferior posición económica se va a sentir más. Estamos muy lejos de que exista en el Perú un sentimiento fuerte de igualdad al cual corresponda un trato igualitario, democrático, y por tanto, ausencia de discriminación (1992, p. 187).

En suma, para Portocarrero, la «utopía del blanqueamiento» es un dispositivo que funda subjetividades escindidas y una comunidad bastante disconforme consigo misma. Produce un universo social donde muchos se avergüenzan de lo propio y están asediados por algo —una herencia— de la que buscan salir. La consecuencia es que desde ahí emerge "un sujeto que no tiene el reconocimiento al que aspira pero que, a manera de compensación, puede imaginar un proceso de depuración y limpieza» (2013, p. 72).

Se trató, en suma, de insistir que la raza todavía activa un conjunto de miedos, tormentos y fantasías emocionales que, como hemos visto, dan cuenta de la colonización del imaginario. Al respecto, fue enfático en proponer que uno de los rasgos distintivos del racismo peruano es que no se trata de una práctica que regula las formas de relación con el otro, sino que además se constituye como «una relación de uno consigo mismo» (Portocarrero, 1992; Zavala y Zariquiey, 2007).

\section{RESISTENCIAS Y CONCLUSIONES}

Portocarrero fue un sociólogo que siempre prestó mucha atención a las dinámicas del arte peruano. Para él, el arte era un hecho social de mucha importancia y lo concibió como una praxis encargada de abrir a la comunidad hacia nuevas figuraciones de sí misma. En su opinión, son las prácticas del arte las que le «devuelven a su sociedad un retrato de sí misma que ella no quisiera ver ni asumir» (2015a, p. 43).

No es este el lugar para estudiar las respuestas artísticas hacia estos imaginarios, pero podemos decir que en las acuarelas de Pancho Fierro, en la obra de Mariátegui, en la narrativa de José María Arguedas y en las actuales pinturas de Claudia Coca (donde observó su articulación con la problemática de género), Portocarrero vio, en efecto, prácticas que se salían de las representaciones dominantes e intentaban instituir un nuevo imaginario nacional. Por ejemplo, sobre las pinturas de Claudia Coca afirmó lo siguiente:

La obra artística de Claudia Coca debe entenderse como reacción a este trasfondo histórico. El vector que la anima es la integración de lo escindido por las alienantes 
demandas del colonialismo. El proyecto es revalorar lo mestizo y luchar por un cambio de sensibilidad estética que pasa por la reivindicación de lo indígena y la liberación del hechizo que lo blanco ejerce sobre nuestros gustos. Es decir por el desaprendizaje del racismo. La revaloración de lo mestizo es también la revaloración de lo femenino. Son como dos frentes en los que la democracia tiene que batallar contras las prácticas que jerarquizan y discriminan (2015b, p. 48).

Las consecuencias de racismo son muchas, pero, en su visión, podemos enumerar las siguientes: la primera es que se trata de una práctica que se ha ido reproduciendo de generación en generación. El racismo está constituido por hábitos de naturaleza colonial que recorren el tejido social y las mentalidades individuales y colectivas. De un lado, constató que vivimos en un país sin entidades colectivas que amparen a los individuos y, de otro, que mucha gente no se da cuenta de sus prácticas racistas porque el racismo actúa como una especie de automatismo que se ha ido sedimentando, desde la nińez, a partir de prejuicios y discursos naturalizadores. Como hemos visto, Portocarrero siempre sostuvo que el racismo porta una dimensión inconsciente y por eso se hace tan difícil de erradicar. El deseo hacia lo blanco no refleja sino una cultura permanentemente acosada por los fantasmas de su pasado:

¿Por qué subsiste? Debe haber varios factores involucrados pero nos referiremos a dos. El primero es la inercia; las mentalidades se heredan y el cambio es lento y difícil. Los padres tienden a reproducir espontáneamente la educación que recibieron. El segundo tiene que ver con el uso más o menos consciente del racismo como un mecanismo de segregación social, de eliminación de un cierto riesgo de competencia invalidando al otro por ser demasiado oscuro y supuestamente feo. Se mantiene así una barrera a la movilidad social. Al respecto es un hecho conocido que en muchas instituciones de nuestro país las posibilidades de acceso y promoción están muy influidas por la apariencia física. Conforme uno se aleja de la base social para acercarse al vértice de las clases altas, el color promedio se va tornando más blanco y menos cobrizo. El hecho colonial no acaba de ser superado y los rasgos físicos siguen tendiendo profundas resonancias (2007, p. 203).

La segunda consecuencia del racismo refiere al profundo descrédito de las figuras de autoridad en el Perú. Día tras día, constatamos un deficit de legitimidad de las autoridades y de las instituciones nacionales. Para los peruanos, en efecto, ninguna autoridad ofrece garantías. Quienes ocupan los cargos se creen «superiores» y quieren demostrar su potencia de alguna manera. Portocarrero sostuvo que este descrédito de la autoridad tiene que ver con una visión muy jerarquizada del orden social que, de manera sorprendente, persiste a la crisis de la relación "patrón-siervo" y que empalma con la falta de cristalización de una relación moderna de «representante-ciudadano». Por eso, en su evaluación sobre los años de Velasco sostuvo que sus reformas «no combatían tanto con la oligarquía, sino con los conflictos 
irresueltos de la sociedad peruana, con la fragmentación y la ingobernabilidad de propias de un sistema de autoridad, donde no hay un sentimiento de autoridad que facilite la confianza» (2003, p. 247).

El debilitamiento de colonialismo no ha seguido tanto una afirmación de la ley y la ciudadanía como una democratización de la trasgresión y de la viveza y de las correspondientes formas de acción colectiva: la mafia y el populismo. Surge entonces escenario de una sociedad de cómplices donde predominan comportamientos individualistas y trasgresores (2015a, p. 331).

La tercera consecuencia refiere a la constatación de que, en el mundo actual, lo indígena solo está valorado como forma de marketing y de diferencia mercantilizada. «En este aspecto la globalización viene a dar un nuevo aire al colonialismo, a la utopía del blanqueamiento» sostuvo enfáticamente (2013, p. 85). Para el mercado neoliberal sigue dominando una estética blanca donde «la especificidad del presente queda desdibujada por los fantasmas del pasado. Y esos fantasmas impiden un diálogo en el que se esclarezca lo nuevo de la situación de hoy» (2010a, p. 17).

Sin embargo, a Portocarrero también le resultaba claro que hoy podemos observar una suerte de lucha entre una creciente demanda de igualdad y la inercia histórica hacia continuar produciendo exclusiones de todo tipo. En ese sentido, sostuvo que existe en el país una especie de "pacto» en el que la discriminación nunca se traduce en una normatividad explícita pero que sigue existiendo como práctica y como «fundamento invisible». En su opinión, el racismo se ha vuelto un fenómeno muy complejo por su poca capacidad predictiva y porque las identidades (entre los discriminadores y discriminados) se confunden y se diluyen en muchos contextos. Sostuvo entonces que, como modelo de interacción con el otro, el racismo es un fenómeno que se reconfigura en el tiempo, que siempre va desplazando su puntería y va modificando las formas en la que ejerce poder a partir de nuevas formas de categorización social. Portocarrero, en suma, entendió el racismo como un fenómeno histórico, pero también como un conjunto de prácticas que siempre están encontrando nuevas estrategias de funcionamiento. Su opción por pensar en la continuidad del racismo le permitió observar sus estrategias para permanecer y para renovarse en nuevos contextos.

Sin embargo, en sus últimos escritos observó cómo viejos componentes culturales se encuentran en permanente negociación bajo nuevos procesos de democratización social que, con todas sus contradicciones, intentan abrirse paso en el Perú de hoy. Pensar esas contradicciones, vale decir, pensar en todo lo que cambia, y en aquello que no, es la posta que dejó para la investigación futura. Hoy, en efecto, sabemos que la subjetividad puede ocupar varias posiciones a la vez y que se trata de una simultaneidad entre lo que se hereda y ha ido retrocediendo en su interacción con nuevas configuraciones sociales. Portocarrero sostuvo que en el Perú actual «sobre- 
viven imágenes que son en gran medida inadecuadas, que ya no corresponden a realidades definidas» porque los cambios sociales van más rápido que las representaciones colectivas (2007, p. 180). En ese sentido, constató que, efectivamente, «el orden colonial fue transformado molecularmente por el mestizaje y la movilidad social; no obstante, el viejo sistema clasificatorio y el espíritu racista habrían subsistido" (2007, p. 180).

Concluyamos de esta manera: es cierto que la sociedad peruana experimenta importantes procesos de democratización social, pero es también verdadero que el racismo siempre se reinventa a sí mismo y se desplaza hacia nuevas prácticas, categorías y formas de clasificación que continúan discriminando e imposibilitando la construcción de una sociedad igualitaria y democrática. Sin duda, es claro que la movilidad social va ganando significativos espacios en el Perú, pero resulta claro que a lo económico todavía le cuesta desprenderse de la pesada carga cultural asociada a lo que se entiende por «raza». Aunque la raza no deja de ser una suerte de «ilusión óptica creada por la pobreza o prosperidad» (2007, p. 179), el gran problema es que «sacudirse de la servidumbre se ha convertido en el Perú en una hazaña individual más que en una empresa colectiva» (2015a, p. 327). En ese sentido, Gonzalo Portocarrero fue terco en afirmar que «la lucha por descolonizarnos continúa todavía» (2013, p. 91).

\section{REFERENCIAS BIBLIOGRÁFICAS}

Drinot, P. (2014). Una vana pretensión: negar el racismo en el Perú. En G. Rochabrún, P. Drinot, y N. Manrique, Racismo. ¿Solo un juego de palabras? (pp. 30-47). Lima: Ministerio de Cultura, IEP.

Manrique, N. (2014). Racismo: una mala palabra. Acerca de un texto de Guillermo Rochabrún. En G. Rochabrún, P. Drinot, y N. Manrique, Racismo. ¿Solo un juego de palabras? (pp. 49-85). Lima: Ministerio de Cultura, IEP.

Mbembe, A. (2016). Crítica de la razón negra. Buenos Aires: Ned ediciones.

Portocarrero, G. (1992). Discriminación social y racismo en el Perú. En N. Manrique, L. Glave y F. Muñoz (eds.), 500 años después... el fin de la historia (pp. 179-197). Lima: Escuela para el Desarrollo.

Portocarrero, G. (1995). El fundamento invisible: función y lugar de las ideas racistas en la República Aristocrática. En A. Panfichi yF. Portocarrero (eds.), Mundos interiores: Lima 1850-1950 (pp. 219-259). Lima: CIUP.

Portocarrero, G. (2003). Memorias del velasquismo. En M. Hamann, S. López, G. Portocarrero y V.Vich (eds.), Batallas por la memoria: antagonismos de la promesa peruana (pp. 229-255). Lima: Red para el Desarrollo de las Ciencias Sociales en el Perú.

Portocarrero, G. (2007). Racismo y mestizaje, y otros ensayos. Lima: Fondo Editorial del Congreso del Perú. 
Portocarrero, G. (2010a). Los fantasmas del patrón y del siervo como desestabilizadores de la autoridad legal en la sociedad peruana. En G. Portocarrero, J. Ubilluz, V.Vich (eds.), Cultura politica en el Perú. Tradición autoritaria y democratizaciónanómica. Lima: Red para el Desarrollo de las Ciencias Sociales.

Portocarrero, G. (2010b). El (des)orden social peruano. En Oido en el silencio. Ensayos de crítica cultural. Lima: Red para el Desarrollo de las Ciencias Sociales en el Perú.

Portocarrero, G. (2013). La utopía del blanqueamiento y la lucha por el mestizaje. En G. Portocarrero, Sombras coloniales y globalización en el Perú de hoy (pp. 165-200). Lima: Red para el Desarrollo de las Ciencias Sociales en el Perú.

Portocarrero, G. (2015a). La urgencia por decirnosotros. Los intelectualesy la idea de nación en el Perú republicano. Lima: PUCP.

Portocarrero, G. (2015b). Imaginando al Perú. Búsquedas desde lo andino en arte y literatura. Lima: PUCP.

Quijano, A. (2000). Colonialidad del poder, eurocentrismo y América Latina. En La colonialidad del saber:perspectivas latinoamericanas (pp. 777-832). Buenos Aires: Clacso.

Rochabrún, G. (2014). Una vana pretensión: ser racista en el Perú. En G. Rochabrún, P. Drinot, y N. Manrique, Racismo. ¿Solo un juego de palabras? (pp. 13-28). Lima: Ministerio de Cultura, IEP.

Rochabrún, G., Drinot, P. y Manrique, N. (2014). Racismo. ¿Solo un juego de palabras? Lima: Ministerio de Cultura, IEP.

Ruiz-Bravo, P.y Neyra, E. (2001). Enfrentados al patrón: una aproximación al estudio de las masculinidades en el medio rural peruano. En S. López Maguińa, G. Portocarrero, R. Silva Santisteban y V. Vich (eds.), Estudios culturales. Discursos, poderes, pulsiones (pp. 211-232) . Lima: Red para el Desarrollo de las Ciencias Sociales en el Perú.

Wetherell, M.y Potter,J. (1992). Mappingthe Language ofRacism. Discourse and the Legitimation of Exploitation. Nueva York: Columbia University Press.

Zavala, V. y Zariquiey, R. (2007). Yo te segrego a ti porque tu falta de educación me ofende: una aproximación al discurso racista en el Perú contemporáneo. En T.Van Dijk (ed.), Discurso y racismo en América Latina (pp. 333-369). Barcelona: Gedisa.

Zavala, V. y Back, M. (2017). Racismo y lenguaje. Lima: PUCP. 